\title{
Elections fédérales 2011
}

Le 23 octobre prochain, le peuple suisse élira ses représentants au Parlement pour les quatre prochaines années. La FMH, acteur important du système de santé et organisation professionnelle regroupant plus de 35000 membres, tient à ce que le corps médical soit représenté aux Chambres et qu'il puisse participer activement aux débats politiques et aux procédures législatives. Nous avons le plaisir de vous présenter dans ces colonnes les candidates et candidats au Conseil national et au Conseil des Etats.

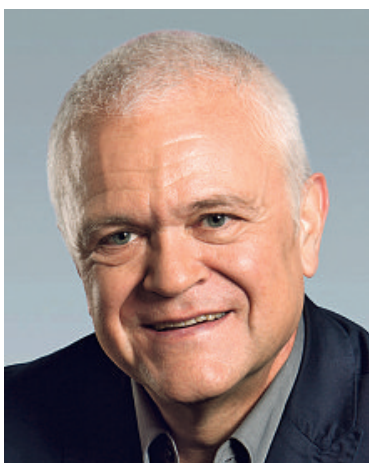

Jürg Knuchel, 1959, Aarau

- Nationalratskandidat SP

- Facharzt FMH für Innere Medizin und Gastroenterologie

- Leitender Arzt für Gastroenterologie und Hepatologie am Kantonsspital Aarau, Einwohnerrat Aarau seit 2009

- Mitglied des kantonalen und Zentralvorstandes des VSAO 1988-1997

- Keine Interessenbindungen

- www.juerg-knuchel.ch

- juerg.knuchel@bluewin.ch
Die zunehmende Bedeutung von Ökonomie und Prozessqualität im Gesundheitswesen erfordert in den nächsten Jahren unsere volle Aufmerksamkeit, damit wir eine Zweiklassenmedizin verhindern und gleichzeitig die zentralen, zutiefst menschlichen Aspekte der Medizin und eine optimale, medizinische Versorgung für alle uneingeschränkt erhalten können. Dafür möchte ich mich auf allen Ebenen einsetzen und damit zum Brückenschlag zwischen Grundversorgern und Spezialisten, zwischen Ökonomie und Medizin beitragen - zum Wohl und zum Vorteil unserer Patienten und damit letztlich von uns allen!

\section{Aargau}

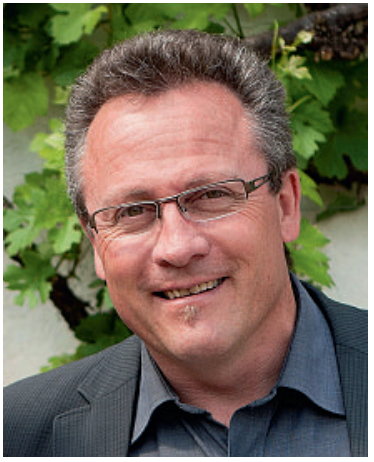

Berne

\section{Daniel Beutler-Hohenberger, 1962, Mühlethurnen}

- Candidat pour le Conseil national UDF

- Médecine générale FMH, médecine manuelle SAMM

- Médecin de famille avec propre cabinet, publicitaire

- Co-président de l'Association faîtière pour une politique de la drogue basée sur l'abstinence, membre du comité référendaire «Révision partielle de la LStup», membre du comité d'initiative «Financer I'avortement est une affaire privée»

- Ancien secrétaire général de l'Association des médecins évangéliques suisses (AGEAS)

- Aucun lien d'intérêts

- www.daniel-beutler-hohenberger.ch

- dan.beutler@hin.ch

Pour un système de santé financièrement supportable, notamment en encourageant la médecine de premier recours, pour une politique de la santé rai- sonnable avec un catalogue de prestations de base restrictif, pour un emploi différencié des prestations médicales et pour davantage de responsabilité personnelle.

Pour un droit à la vie étendu au commencement de la vie et à la fin, basé sur la dignité indivisible de toutes les vies humaines, soit une entière protection de la vie en devenir avec des limites claires pour la procréation médicalement assistée et une interdiction de l'euthanasie active et de l'assistance au suicide.

Pour une politique de la drogue basée sur l'abstinence avec maintien et encouragement des thérapies basées sur l'abstinence et un concept de traitement durable pour tous les toxicomanes. Contre l'élargissement des traitements de substitution et contre toutes les tentatives de légalisation. 


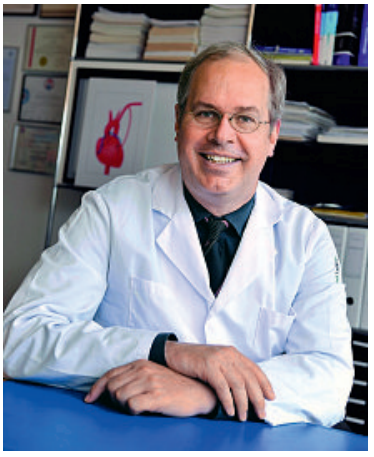

Berne

\section{Thierry P. Carrel, 1960, Berne}

- Candidat pour le Conseil national PLR

- Chirurgie générale FMH, Chirurgie cardiaque et vasculaire thoracique FMH, Schwerpunkt Chirurgie vasculaire

- Directeur de la clinique universitaire de chirurgie cardiovasculaire, Hôpital de l'Ile

- Président de la Société suisse de chirurgie cardiaque et vasculaire thoracique (2011-2012), Secrétaire de la Société suisse de chirurgie thoracique et cardiovasculaire (1996-2010), Représentant de la société à la Chambre médicale et à la CFPC (actuel ISFM) depuis 1996, Représentant de la discipline à la Commission des titres depuis 1999

- Aucun lien d'intérêts

- www.thierry-carrel.ch

- thierry.carrel@insel.ch

La planification cantonale dans le domaine hospitalier doit se limiter au strict nécessaire. La médecine ne doit en aucun cas devenir étatique. Dans le domaine de la santé la stratégie doit être élaborée en régions et non plus en cantons. Le libre choix du médecin et de l'hôpital doit être garanti sans restrictions.

Le numerus clausus doit impérativement être assoupli et de nouvelles places de formation créées.Il ne manque pas seulement des médecins généralistes mais de plus en plus aussi de spécialistes. Le choix des futurs médecins ne doit pas être basé sur des examens psychologiques douteux mais bien plus sur le savoir, la motivation, l'aptitude au dialogue et l'empathie.

Il reste encore beaucoup à faire pour trouver des solutions aux problèmes éthiques brûlants (le diagnostic pré-implantation, le don d'organes et l'aide au suicide) sans oublier l'amélioration des conditions de travail des professions de la santé sans vouloir académiser à outrance le personnel soignant.

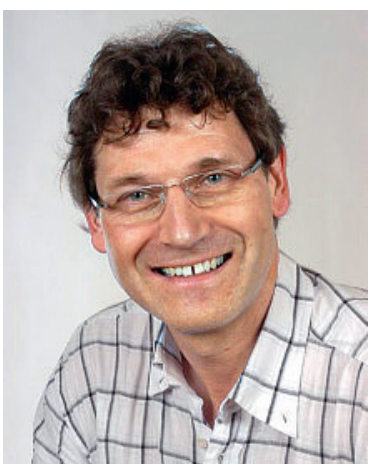

Berne

\section{Willfried Gasser, 1957, Wabern}

- Candidat pour le Conseil national PEV

- Dr méd., pas de titre de spécialiste

- Consultant, conseiller en entreprise

- Membre du Grand Conseil du canton de Berne de 2005 à 2010, membre de la Commission de haute surveillance

- Président de l'Alliance évangélique suisse

- www.wilfgasser.ch

- wi.gasser@bluewin.ch

Les incitatifs adéquats doivent être créés au plan national afin de garantir une couverture médicale de bonne qualité et d'un coût raisonnable. Les différences en matière de financement entre le secteur ambulatoire et le secteur stationnaire doivent être supprimées, car elles ne font aucun sens dans le cadre d'un système de soins intégrés. Je tiens à une concurrence juste (égalité des chances) et forte entre les hôpitaux privés et publics. J'estime que la concurrence entre les caisses-maladie ne permet pas vraiment de limiter la hausse des coûts et je soutiens donc l'idée d'une caisse unique.

Sur le plan cantonal, j'ai déposé en 2010 une motion demandant la restauration du Stadtspital sur le site de l'Ile. Il est nécessaire de réformer la structure et de disposer pour ce faire d'une planification cantonale claire. La loi sur les soins hospitaliers doit être remplacée à moyen terme par une loi sur les soins de santé.

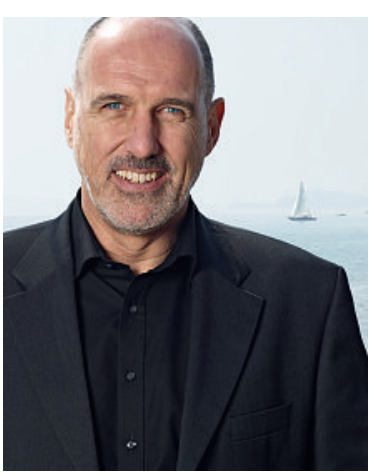

Berne

\section{Jacques de Haller, 1952, Berne}

- Candidat pour le Conseil national PS

- Médecine générale FMH

- Président de la FMH

- Président de la SSMG (1997-2004); divers autres mandats de politique professionnelle

- Aucun lien d'intérêts

- www.jacquesdehaller.ch

_ jdh@jacquesdehaller.ch, jdh@fmh.ch
J'ai décidé d'être candidat au Conseil national pour mettre à profit mon expérience le mieux possible: je connais bien les difficultés des médecins, et j'ai des réseaux denses et utiles - ce sera un avantage décisif pour la FMH d'être présent là-même où les décisions sont prises.

Notre système de santé, pour qu'il soit juste et efficace, doit garantir l'accès de toutes et tous aux soins qui leur sont nécessaires, au même haut niveau de qualité. Et cela demande notamment des professionnel-les bien formé-es, bénéficiant de conditions de travail adéquates et d'une bonne qualité de vie.

C'est pour cela que je m'engage. 


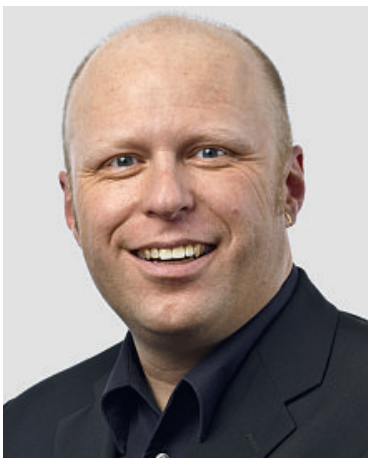

Berne

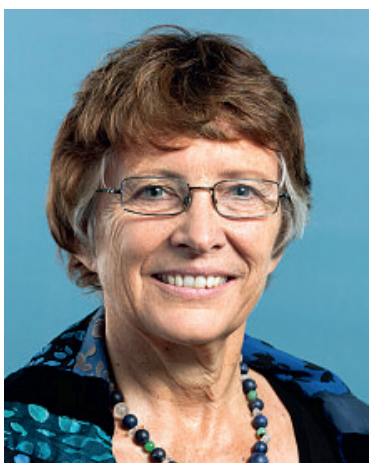

Berne
Dieter Hötsch, 1964, Urtenen-Schönbühl

- Candidat pour le Conseil national Partie des vert'libéraux

- Chirurgie orthopédique FMH

- Conseil et expertise en médecine d'assurance

- Membre de la commission Honoraires et tarifs de la SSOT depuis 2002

- Aucun lien d'intérets

- www.be.grunliberale.ch

- hoetsch@intergga.ch

Issu d'une discipline chirurgical, et en contact avec différents acteurs du domaine de la santé dans le cadre de ma fonction de médecin d'assurance, il m'ar- rive malheureusement encore d'être régulièrement témoin d'un manque de compréhension mutuelle, notamment entre les différentes disciplines de notre profession. C'est pour cette raison que, dans l'intérêt d'un niveau élevé de prise en charge médicale de notre population, mon objectif principal en politique de santé est de créer une atmosphère constructive en vue de réunir les différents groupes autour d'une table pour discuter des problèmes à relever et trouver des solutions acceptables. Je ferai tout pour que nous, les médecins, soyons de nouveau considérés comme des partenaires compétents, à même de collaborer dans un esprit consensuel à l'avenir de notre système social et de santé.

\section{Danielle Lemann, 1950, Langnau}

- Candidate pour le Conseil national PS

- Médecine générale FMH

- Médecin de famille dans un cabinet de groupe, médecin à temps partiel à I'hôpital régional de I'Emmental

- Membre du Grand Conseil du canton de Berne

- Experte en médecine complémentaire auprès de la Commission fédérale des médicaments, déléguée de la Société des médecins du canton de Berne, membre du comité directeur de l'Association suisse des médecins d'orientation anthroposophique

- Lien d'intérêts: Médecins en faveur de l'environnement, CAS, SolidarMed, PSR

- www.danielle-lemann.ch

- dlemann@hin.ch

- Je m'engage pour un réel renforcement de la médecine de famille: il faut enfin que les positions du TARMED relatives aux médecins de premier re- cours soient justes, éviter les expressions dénuées de sens telles que le Managed care, mais continuer à encourager la coordination entre les acteurs du système de santé et augmenter le nombre de professeurs enseignant la médecine de famille.

- Introduction consciencieuse des forfaits par cas liés au diagnostic après avoir réglé les problèmes encore en suspens comme la protection des données, la formation universitaire et postgraduée des médecins, le traitement de patients polymorbides, palliatifs et pédiatriques, et la prise en compte de la charge des soins.

- Mise en œuvre du nouvel article constitutionnel relatif aux médecines complémentaires et de ses cinq exigences fondamentales.

- Mise en œuvre des demandes des médecins en faveur de l'environnement et des médecins pour une responsabilité sociale.

- Renforcement de la médecine palliative et de la conscience de notre propre mortalité.

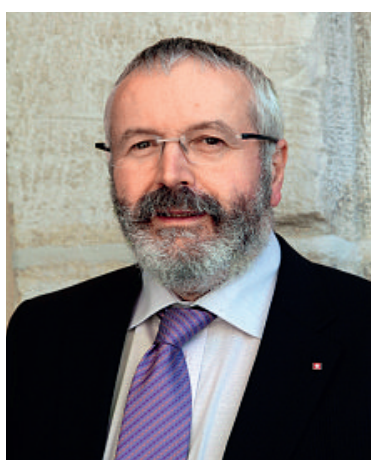

Jura

\section{Dominique Baettig, 1953, Delémont}

- Candidat pour le Conseil national UDC

- Psychiatrie FMH

- Pratique libérale

- Conseil national (2007-2011), Conseiller de Ville Delémont (2004-2012)

- Aucun lien d'intérêts

- www.udc-jura.ch

- dominiqueb@bluewin.ch

Aujourd'hui, l'enjeu politique n'est plus entre une gauche sociale et une droite économique, mais entre les partisans et les résistants à la mondialisation.
Entre une oligarchie financière et déracinée et les citoyens enracinés, rattachés à une culture, une identité, à la démocratie directe de proximité, dans une entité de taille humaine et historique. L'UDC incarne ces valeurs de résistance morale, spirituelle et d'esprit d'entreprise, de passion de libertés.

En tant que médecin attaché aux valeurs non marchandes, je m'inquiète des dérives de la médecine technique, industrielle, de redistribution illusoire et coûteuse d'un capital de soins de santé. Le médecin doit rester un conseiller de confiance, soucieux de ne pas nuire. Les soins gérés sont une dérive de gestion économique dangereuse. 


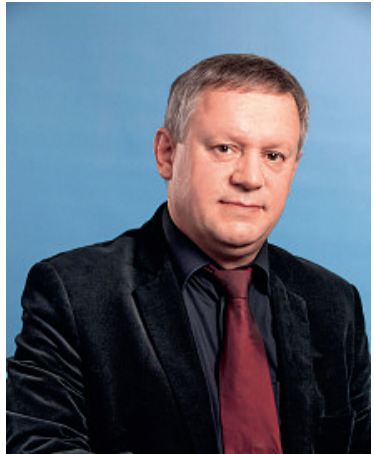

Jura

\section{Pierre-Alain Fridez, 1957, Fontenais}

- Candidat pour le Conseil national PS

- Médecine générale FMH

- Cabinet médical à Fontenais

- Député, ancien maire

- Membre commission politique de santé MFE

- pafmabe@bluewin.ch

Médecin généraliste installé dans un village en Ajoie depuis 23 ans, j'ai assumé en parallèle depuis 15 ans différents mandats politiques, en particulier maire de ma commune durant 12 ans et député pendant 9 ans au sein du Parlement jurassien.
Mes motivations pour briguer un siège au Conseil national résident bien entendu dans la défense des valeurs qui m'ont fait adhérer au parti socialiste: justice et protection sociale, défense de l'environnement, égalité des chances notamment, mais également dans mon engagement prioritaire dans le domaine de la politique de la santé: combat en faveur d'une caisse maladie unique, reconnaissance et renforcement du statut des médecins de premier recours, prévention et promotion de la santé, égalité d'accès aux soins.

Je siège au sein de la Commission politique de l'association suisse des médecins de famille MFE.

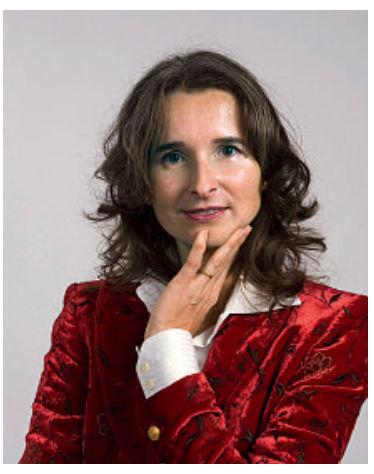

\section{Yvette Estermann, 1967, Kriens}

- Nationalratskandidatin SVP

- Dr. med., Zusatz-Ausbildung in klass. Homöopathie bei SVHA und SAHP

- Beraterin in eigener Firma: «Estermann life coaching»

- Kantonsrätin 2005-2007, Nationalrätin ab 2007, Mitglied der Kommission für soziale Sicherheit und Gesundheit NR (SGK-NR)

- Mitglied bei VSAO und SAHP

- www.yvette-estermann.ch und www.estermannaktuell.ch

- info@yvette-estermann.ch

\section{Luzern}

Ob früher als Kantonsrätin, Präsidentin der Gesundheitskommission im kantonalen Parlament oder jetzt auch als Nationalrätin und Mitglied der SGK-NR setze ich mich für ein Gesundheitssystem ein, welches den Bedürfnissen unserer Bevölkerung entspricht. Dies muss aber bezahlbar sein und möglichst frei von Missbrauchsmöglichkeiten. Als Ärztin weiss ich, was für eine wichtige und verantwortungsvolle Aufgabe in unserer Gesellschaft die Ärztinnen und Ärzte erfüllen. In diesem Sinne habe ich immer bei politischen Anliegen gehandelt und gedenke, dies auch weiterhin zu tun. Ich bin überzeugt, dass alle Ärztinnen und Ärzte sowie ihre Mitarbeiter im Gesundheitssektor immer nach ihrem besten Wissen und Gewissen handeln, - im Dienst der Bevölkerung. Diese Bemühungen unterstütze ich gern, - mit Überzeugung!

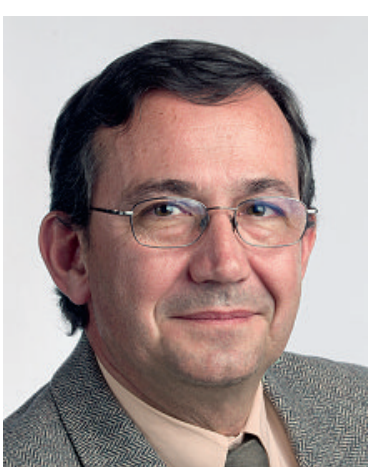

\section{Blaise Courvoisier, 1955, La Chaux-de- Fonds}

- Candidat pour le Conseil des Etats UDC

- Chirurgie FMH

- Indépendant en cabinet et clinique privée

- Député au Grand Conseil neuchâtelois,

- Président commission santé du Grand Conseil

- Président du Conseil d'Administration Clinique de la Tour

- www.udc.ne

- b.courvoisier@bluewin.ch
La santé et son enseignement doivent être régionalisés, au détriment de la cantonalisation actuelle. Il faut avoir un réseau d'hôpitaux régionaux de proximité pour les soins de base et des centres universitaires régionalisés et redimensionnés pour les pathologies les plus graves. La médecine doit être enseignée dans ces centres régionaux avec la possibilité pour un nombre restreint d'étudiants de poursuivre en milieu universitaire.

\section{Neuchâtel}




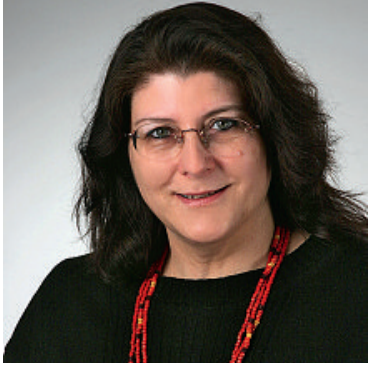

St. Gallen
Veronika Breitler-Voigt, 1957, Kirchberg

- Nationalratskandidatin BDP

- Dr. med., Dipl.-Psych., FMH Psychiatrie/Psychotherapie, Fachärztin für Psychosomatische Medizin, SAPPM Psychosoziale Medizin, Systemische Familientherapie, Psychoanalyse, Gruppenanalyse SGAZ (Zürich und London)

- Niedergelassen in eigener Praxis

- Keine Interessenbindungen

- www.ostschweizerinnen.c und www.bdp-sg.ch

- www.drbreitler.ch

- veronika.breitler@tbwil.ch
Dafür engagiere ich mich in der BDP:

- Gesundheitspolitik (u.a. mehr Eigenverantwortung der Bevölkerung für die eigene Gesundheit);

- Sozialwerke (sinnvolle Wiedereingliederung nach IV);

- Frauenfragen (Gender, Vereinbarkeit von Karriere und Lifebalance);

- Familienpolitik, Bildung, öffentliche Sicherheit.

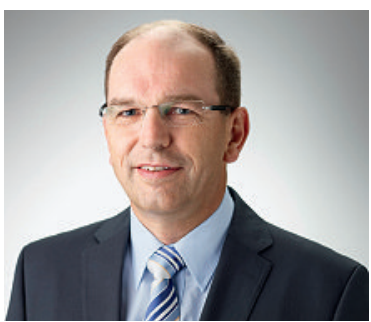

\section{Bruno Damann, 1957, Gossau}

- Nationalratskandidat CVP

- Allgemeinmedizin FMH

_ Praxistätigkeit im Ärztehaus Gossau

- Parlamentarier der Stadt Gossau

- Interessenbindungen: Adviser $\mathrm{HCl}$ Galenica

- www.Bruno-Damann.ch

- bdamann@hin.ch, Bruno.Damann@bigfoot.com
Ich vertrete die freie Arzttätigkeit. Für mich ist es wichtig, dass die Grundversorger gestärkt werden. Freier Zugang zur Medizin für alle. Der staatliche Einfluss auf die Medizin darf nicht noch mehr zunehmen. Hausarztmodelle ausbauen. Spitalplanung über die Kantonsgrenze hinaus. Keine ambulanten Fallpauschalen.

\section{St. Gallen}

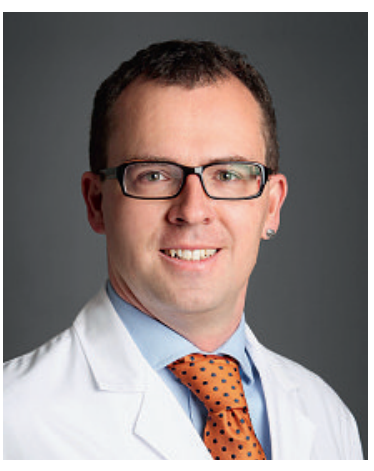

St. Gallen

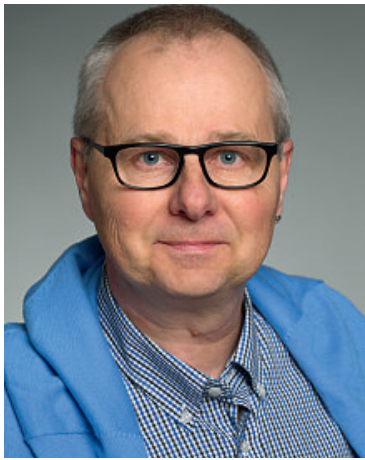

Thurgau

\section{Friedrich von Toggenburg, 1974, Buchs}

- Nationalratskandidat CVP

- Urologie / operative Urologie

- Praxisarzt

- Keine Interessenbindungen

- www.friedrich-vontoggenburg.ch

- friedrich.vontoggenburg@hin.ch

Die Einführung der DRG ist beschlossen und kann, realistisch betrachtet, nicht mehr aufgeschoben werden. Um die negativen Auswirkungen dieses Systemwechsels möglichst bald zu eliminieren, ist eine intensive Begleitforschung notwendig. Dies muss insbesondere auch von parlamentarischer Seite gefordert werden.

Die Problematik der unzureichenden Anzahl medizinischer Ausbildungsplätze muss schnell gelöst werden. Wir können uns nicht erlauben, dass weiterhin mehr als ein Viertel der benötigten Ärzte aus dem Ausland angeworben werden müssen.

Der stetige Anstieg der OKP-Prämien kann langfristig nur durch eine Straffung des OKP-Kataloges gebremst werden. Dieses ethisch wie politisch schwierige Thema muss bald, aber breit abgestützt angegangen werden.

\section{Bernhard Wälti, 1956, Freidorf}

- Nationalratskandidat SP

- Allgemeinmedizin FMH

- Selbständig als Landarzt

- Kantonsrat Thurgau seit 2005

- ehem. Vorstandsmitglied VSAO St. Gallen; seit 1995 Lehrbetrieb für MPA

- Präsident Energiegenossenschaft Roggwil, Beirat Thurgauer Programm zur Brustkrebsfrüherkennung, Mitglied Ärztegesellschaft Thurgau, Mitglied Pro Natura Thurgau

- www.worthalten.ch

- bmwaelti@hin.ch
Die bizarre Bürokratie muss fallen!

Andere haben ihr Gesundheitswesen ruiniert. Lernen wir von ihnen und machen wir nicht die gleichen Fehler! Unser erstklassiges Gesundheitswesen darf nicht von Leuten zerschlagen werden, die davon nichts verstehen, und ohne dass wir Besseres zur Verfügung haben. Deshalb brauchen wir mehr Fachwissen und Unvoreingenommenheit in der Politik, nicht Ideologie. Denn Medizin kennt keine Partei, sondern Vernunft.

Wenn wir die Stärke der Schweiz bewahren wollen, müssen wir ihren Zusammenhalt erhalten. Dazu gehört, dass Medizin bezahlbar ist. Wir Ärzte - Hausärzte wie Spezialisten - leisten erstklassige Arbeit. Dafür wollen wir faire Bedingungen, keine bizarre Bürokratie. Und wir wollen Patienten mit Eigenverantwortung, gezielte Forschung, ausreichend Studienplätze. Eine clevere Medizin eben. 


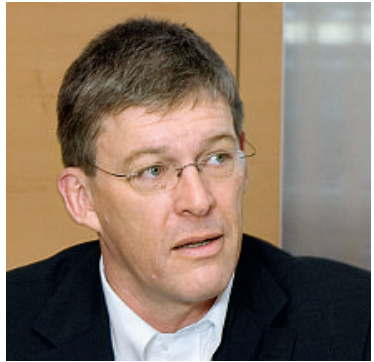

Thurgau

\section{Mathias Wenger, 1956, Häuslenen}

- Nationalratskandidat CVP

- FMH Innere Medizin

- Kantonsarzt Kt. Thurgau zu 85\%,

Vertrauensarzt Bundesamt für Zivilluftfahrt und Bundesamt für Verkehr zu 15\% (1991-2007

Hausarzt; 2007-2010 teilzeitlich Geschäftsführer Schlossberg Ärztezentrum Frauenfeld, kleines Pensum als Hausarzt und Vertrauensarzt, 50\% stellvertretender Kantonsarzt Thurgau)

- 1986-1988: Vizepräsident VSAO, Sektion Thurgau, 1992-2000 Vorstandsmitglied Ärztegesellschaft Thurgau, 2000-2005: Präsident Ärztegesellschaft Thurgau, 2004-2005 Co-Präsident VEDAG, seit 2005 bis heute Vorstandsmitglied VEDAG, 2008 bis 2010: Vorstandmitglied SGARM

- Interessenbindungen: Vorstandsmitglied VEDAG, Kantonsarzt, Vertrauensarzt BAZL, BAV

- www.mathias.wenger.ch

- mathias.wenger@hin.ch
Dafür engagiere ich mich:

- Chancengerechte, medizinische Versorgung und Finanzierung, d. h. Eigenverantwortung, Solidarität, Subsidiarität;

- Vernetzung zwischen Grundversorgern, Spezialisten und Spitälern über die gesamte Behandlungskette, mit Einbezug der Pflege und des Sozialwesens;

- Förderung des Nachwuchses in Pflege und ärztlichem Bereich;

- Förderung der Grundversorgung, der psychischen Gesundheit der Prävention und der Gesundheitsförderung;

- Kostenträger sind keine Leistungsanbieter;

- Korrekte Qualitätsmessung, faire Wirtschaftlichkeitskontrollen, direkte Medikamentenabgabe, adäquate, nicht kantonsabhängige Leistungsabgeltung.

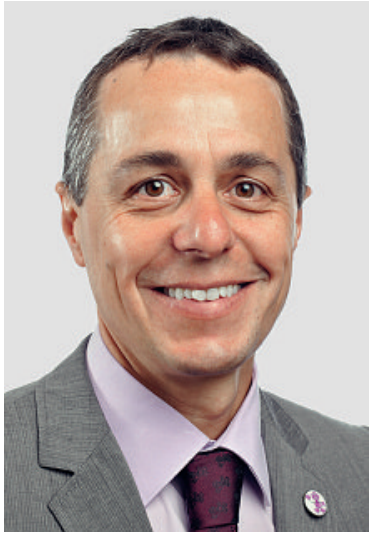

Ticino

\section{Ignazio Cassis, 1961, Collina d'Oro}

- Candidato al Consiglio nazionale PLR

- FMH prevenzione e salute pubblica, FMH medicina interna

- Consigliere Nazionale e Vicepresidente della FMH

- Dal giugno 2007 membro del Consiglio nazionale, dall'aprile 2004 membro del Consiglio comunale

- Dal giugno 2008 Vicepresidente della FMH, 1992-1996 Vicepresidente ASMAC; 1989-1992 Presidente ASMACT

- Presidente della Fondazione EQUAM, Presidente di Fourchette verte Ticino, Membro di comitato del Forum Managed Care

- www.ignaziocassis.ch

- ignazio.cassis@parl.ch
Nella legislatura 2007-11 mi sono impegnato in Parlamento per una nuova Legge federale sulla prevenzione e per migliorare la LAMal. Ho proposto - con successo - una soluzione per impedire alle casse malati di selezionare le persone sane. Mi sono battuto per migliorare il modo di calcolare l'economicità dei medici e per modellare due grosse riforme strutturali: quella sul finanziamento ospedaliero e quella sulle cure integrate. Nella FMH ho stimolato il lavoro in Comitato e negli altri Organi. Sono stato ospite di numerose società mediche cantonali e specialistiche per spiegare il lavoro politico e la sua logica. Desidero essere rieletto in Parlamento perché vorrei proseguire il lavoro proprio ora che ho maggior competenza e legittimazione. Perciò mi permetto di chiedere il vostro sostegno.

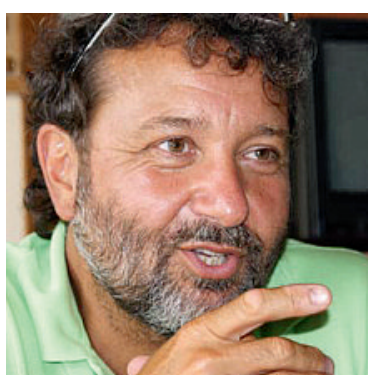

Ticino

\section{Paolo Cipriano Emanuele Peduzzi, 1963, Bellinzona}

- Candidato al Consiglio nazionale PPD

- Pediatra

- Studio medico in proprio a Bellinzona e Medico Scolastico

- Gran Consigliere in carica dal 2010

- Presidente del Trust Center Ticino

- Conflitti d'interessi: Presidente di alcune Associazioni caritative senza lucro

- www.ilpediatra.ch - www.pesovia.ch - www. Con-Tatto.ch

- paolo@ilpediatra.ch
Nato in Ticino da una famiglia che si è sempre interessata di ciò che succede attorno, ho sempre abbinato il mio studio, la mia formazione e ora la mia attività professionale con attività politiche. La politica è un modo di mettersi a servizio degli altri e della comunità. Come nella parabola dei talenti: chi ne ha li metta a disposizione!

La politica sanitaria svizzera è stata stravolta in questi anni e come medici siamo spettatori ignari di capovolgimenti tali nella politica sanitaria da mettere a repentaglio l'arte medica tout-court! Una nostra presa di posizione e una nostra attiva partecipazione alle decisioni di politica sanitaria federale e cantonale non può essere procrastinata, ne va della qualità della medicina stessa e della salute in senso più largo della nostra Nazione e della nostra gente. 


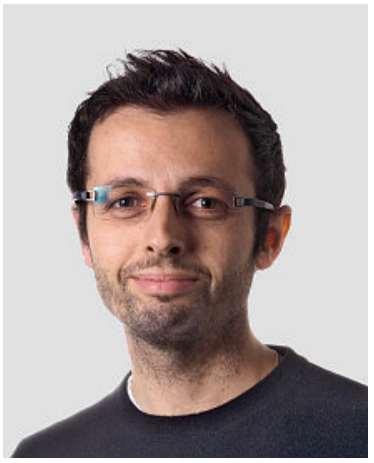

Zürich

\section{David Garcia Nuñez, 1975, Zürich}

- Nationalratskandidat AL

- Psychiatrie und Psychotherapie

- Oberarzt in der Klinik für Psychiatrie und Psychotherapie am UniversitätsSpital Zürich

- Ex-Mitglied der Ständigen Kommission für Weiterund Fortbildungsfragen der SGPP

- Keine Interessenbindungen

- dagar@doctor.com

DRG/Fallpauschalen

- Sofortiges Moratorium, bis Fragen der Begleitforschung, der med. Aus- und Weiterbildung und der Sicherstellung genügend qualifizierter med. Stellen geklärt sind
Stärkung der medizinischen Grundversorgung

- Förderung von Massnahmen, um die qualitativ hausärztliche und psychiatrische Betreuung der Bevölkerung zu gewährleisten.

Besserstellung nicht-invasiver Leistungen

- Um «medizinischen Aktionismus» möglichst zu unterbinden, sollte die Entgeltungskluft zwischen invasiven und nicht-invasiven Interventionen möglichst reduziert werden.

Abschaffung des Numerus clausus

- Notwendige Ressourcen, um genügend Medizinerinnen und Mediziner auszubilden, werden zur Verfügung gestellt. Nur so kann die sich bereits abzeichnende katastrophale Versorgungssituation abgewendet werden.

Einführung einer einkommensabhängigen Krankenkassenfinanzierung.

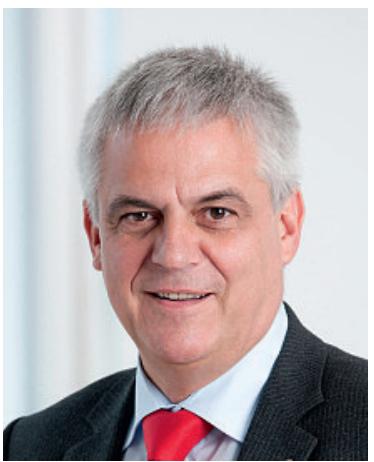

Josef Widler, 1954, Zürich

- Nationsratskandidat CVP

- Allgemeine Medizin FMH

- Hausarzt, Ärztlicher Leiter Ärztehaus Zürich West

- Gemeinderat der Stadt Zürich

- Vizepräsident der Ärztegesellschaft Kanton Zürich

- Keine Interessenbindungen

- www.josef-widler.ch

- josef.widler@bluewin.ch
Die Kosten unseres Gesundheitswesens werden durch strukturelle Mängel unnötig in die Höhe getrieben.

Deshalb setze ich mich ein für

- die Aufhebung des Kontrahierungszwanges,

- die Aufhebung des Tarifschutzes,

- die Vereinfachung des Kosten treibenden Versicherungssystems (KGV, UVG, IV),

- die Einführung einer Einheitskrankenkassenprämie,

- die Abschaffung nicht evidenzbasierter Qualitätskontrollen,

- die Straffung des Leistungskataloges,

- die Zentralisierung der Spitzenmedizin.

\section{Zürich}

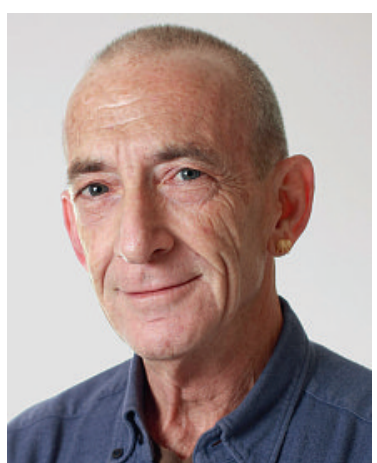

Zürich

\section{David Winizki, 1948, Zürich}

- Nationalratskandidat AL

- Allgemeine Medizin FMH

- Hausarzt

- Keine Interessenbindungen

- david.winizki@hin.ch

Als Hausarzt werde ich oft konfrontiert mit der Verarmung breiter Bevölkerungsschichten, immer knauserigeren Sozialversicherungen, omnipotenteren Krankenkassen und einer zunehmenden Ökonomisierung des Gesundheitswesens. Dieses gehört jedoch zu jenen Gütern, die wir nicht zum Spass konsumieren, sondern zum Überleben brauchen. Es muss deshalb der ganzen Bevölkerung ohne Rationierungen zugänglich sein und solidarischer als heute bezahlt werden.

Budgetverantwortung oder Fallpauschalen implizieren Rationierungen für Grundversicherte und sind deshalb ungerecht. Leistungserbringer sollen gut aus-, weiter- und fortgebildet werden, vernetzt arbeiten und adäquat bezahlt werden. Existenzbedingungen, die ein selbstbestimmtes Leben in Würde erlauben, sind als Gesundheitsvorsorge wichtiger als Birchermüesli und Joggen. 